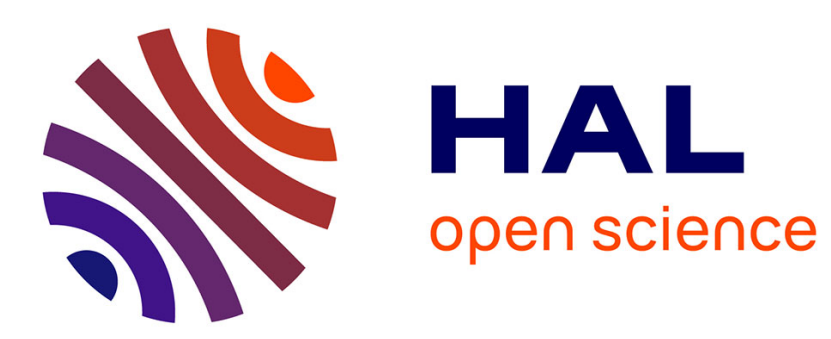

\title{
NLOS-Aware VLC-based Indoor Localization: Algorithm Design and Experimental Validation
} Chuanxi Huang, Xun Zhang, Fen Zhou, Zhan Wang, Lina Shi

\section{To cite this version:}

Chuanxi Huang, Xun Zhang, Fen Zhou, Zhan Wang, Lina Shi. NLOS-Aware VLC-based Indoor Localization: Algorithm Design and Experimental Validation. IEEE Wireless Communications and Networking Conference (WCNC 2020), Apr 2020, Seoul, South Korea. hal-02533000

\section{HAL Id: hal-02533000 https://hal.science/hal-02533000}

Submitted on 6 Apr 2020

HAL is a multi-disciplinary open access archive for the deposit and dissemination of scientific research documents, whether they are published or not. The documents may come from teaching and research institutions in France or abroad, or from public or private research centers.
L'archive ouverte pluridisciplinaire HAL, est destinée au dépôt et à la diffusion de documents scientifiques de niveau recherche, publiés ou non, émanant des établissements d'enseignement et de recherche français ou étrangers, des laboratoires publics ou privés. 


\title{
NLOS-Aware VLC-based Indoor Localization: Algorithm Design and Experimental Validation
}

\author{
Chuanxi Huang, Xun Zhang, Fen Zhou, Zhan Wang, Lina Shi
}

\begin{abstract}
The Visible Light Indoor Positioning System (VLIPS) has been a popular research area recently. In VL-IPS, many localization methods have been proposed by leveraging the Received Signal Strength (RSS) based trilateration. However, the traditional RSS based trilateration localization (RSS-TL) method is very sensitive to the lighting environment and would results in a big localization error due to the presence of nonline-of-sight (NLOS) light signal. In light of this, we propose a novel NLOS-aware localization algorithm, namely Enhanced Fingerprinting-aided RSS-TL (EFP-RSS-TL). It permits to improve the localization accuracy for the corner regions of a room by eliminating the NLOS impact while keeping the same high accuracy for the room center. This is achieved by leveraging a RSS fingerprint database which records the line-of-sight (LOS) light power ratios beforehand. For validation purpose, we built a real VL-IPS platform and implemented the proposed algorithm. Experimental results show that the proposed NLOS-aware EFPRSS-TL algorithm enables to reduce significantly the average positioning error (by up to $79 \%$ ) compared to its counterparts. Besides, our proposal cuts the database size by $50 \%$ and is more robust to environment changes. In a room of $4.7 \mathrm{~m} \times 2.7 \mathrm{~m}$, the achieved average positioning error is around $6 \mathrm{~cm}$ when it is vacant and it is no more than $14.5 \mathrm{~cm}$ when it is occupied by several people.
\end{abstract}

Index Terms-Indoor Positioning System, Visible Light Communications (VLC), Received-Signal-Strength based trilateration

\section{INTRODUCTION}

Visible Light Indoor Positioning System (VL-IPS) has attracted a lot of attentions of researchers recently, because it has many advantages. With the long lifetime of LEDs and the installed illumination systems, the VL-IPS is not only able to provide a high positioning accuracy but also requires low installation \& maintenance cost. Moreover, the visible light has a $10^{4}$ wider bandwidth than radio spectrum and it does not have any electromagnetic interference. Thus, the VL-IPS is suitable for a large number of users and radio frequency (RF) sensitive environments, like hospital and gas station [1].

A VL-IPS locates the target based on visible light communications (VLC) [2,3]. As shown in Fig. 1, it consists of three parts: VL-IPS transmitter (Tx), VL-IPS receiver (Rx) and positioning algorithm. The VL-IPS Txs are installed on the ceiling with known positions and the VL-IPS Rx is carried by the target or mobile users. Positioning Reference Signals

C. Huang, X. Zhang, F. Zhou, Z. Wang, and L. Shi are with the LISITE lab of Institut Supérieur d'Électronique de Paris, France. F. Zhou is also with the CERI-LIA lab at the University of Avignon, France. (emails: fen.zhou@univavignon.fr, firstname.lastname@isep.fr). The work of F. Zhou is partially supported by the open project (2020GZKF017) of the State Key Laboratory of Advanced Optical Communication Systems and Networks, Shanghai Jiao Tong University, China.

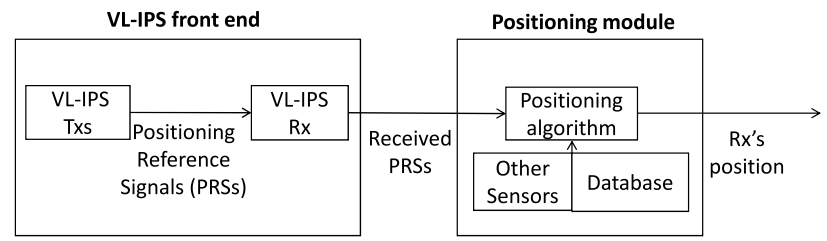

Fig. 1. Overview of VL-IPS.

(PRSs) are emitted by Txs and detected by the Rx. The positioning algorithm will then analyze the received PRSs and calculate the target position.

Many works have been done for VL-IPS. According to a recent survey [4], the positioning algorithms play an important role in VL-IPS and nearly one-third of works focus on them. According to the measurement parameters, these algorithms can be divided into four categories, time measurement algorithms [5] [6], signal strength measurement algorithms [7] [8], signal phase measurement algorithms [9] and others [10] [11]. The Received Signal Strength based Trilateration Localization (RSS-TL) algorithm can achieve a high positioning accuracy with low system complexity [4], it thus becomes more and more popular for VL-IPS. But the RSS-TL has a critical limitation, i.e., the received PRSs powers are sensitive to indoor lighting environment, while a changeable indoor environment is unavoidable. According to the literature [12] [13], the reflection and the presence of non-line-of-sight (NLOS) light is the most important and common impact factor on VL-IPSs for indoor lighting environment, which may cause a significant positioning error with 1 to 2 meters. But there are few effective solutions to reduce this impact. To this end, the Received Signal Strength based Fingerprinting (RSS-FP) is adopted extensively because of its improvement on positioning accuracy [14] [15]. In RSS-FP, the user position estimation is based on a database recording the pre-collected RSS of a set of reference points from the experiment room. In the positioning phase, according to the RSS measured at the mobile target, $\mathrm{K}$ nearest reference points to the mobile target are identified. Then, the position of the mobile target is approximated by a weight average of these $\mathrm{K}$ point coordinates. However, the average positioning accuracy is still not satisfying (in the order of $20 \mathrm{~cm}$ ), and the positioning error can be very large at the room corners. Moreover, this method is still very sensitive to lighting environment changes.

In this paper, we propose an NLOS-aware localization method, namely Enhanced Fingerprint RSS Trilateration (EFP- 
RSS-TL), to improve the positioning accuracy and system robustness. Different from the RSS-FP solution proposed in [14], our algorithm leverages an EFP database storing the line-of-sight light power ratios instead of the absolute value of RSS. With the help of this EFP database and K-Nearest Neighbors (K-NN) method, the impact of NLOS and reflection can be eliminated so that the received Line-of-Sight (LOS) PRSs powers can be estimated as precisely as possible. This in turn enables to cut the the positioning error, especially for the corner regions of a room, where light reflection is significant.

The main contributions of this paper are summarized as follows:

- Through numerical simulations, we show that the light reflection and the presence of NLOS is an important factor degrading the positioning accuracy.

- To improve the positioning accuracy and system robustness, we propose an EFP-RSS-TL method, which leverages an EPF database to eliminate the impact of NLOS and light reflection.

- The proposed solution is validated by building a real VLIPS. Experimental results demonstrate that our solution outperforms its counterparts in terms of positioning accuracy (reducing up to $79 \%$ of the average positioning error) and system robustness. Besides, it requires a smaller storage size.

The rest of this paper is organized as follows. We first analyze the impact of light reflection on localization accuracy in Section II, and then propose an NLOS-aware algorithm to eliminate its impact in Section III. To validate the proposed solution, experiments carried out on a real VL-IPS are presented in Section IV. Finally, we conclude this paper in Section V.

\section{RSS-TL LOCALIZATION SYSTEM AND THE IMPACT OF NLOS SIGNAL}

In this section, we will first present the traditional RSSTL [4], and then analyze the key factors which degrading the positioning accuracy of this algorithm.

In the VL-IPS, several LEDs are used as the transmitters. The target is equipped with a Photodiode (PD), which is served as a receiver and used for localization. The RSS-TL uses the received signal powers of several LEDs to compute the PD position. Let $J$ be the number of LEDs used and at least three LEDs are generally required, $J \geq 3$. The height of the target PD is fixed and known, and the orientation of the PD is assumed to be always vertical to the ceiling. We denote the vertical height between the target PD and the LEDs as $h$, and $m$ is the lambertian factor of an LED. The effective area of a PD is noted as $A_{P D}$. For the $j$-th LED, $P t_{j}$ represent its transmitted PRSs power and $P r_{j}$ signifies the received PRSs power at the target PD from this LED.

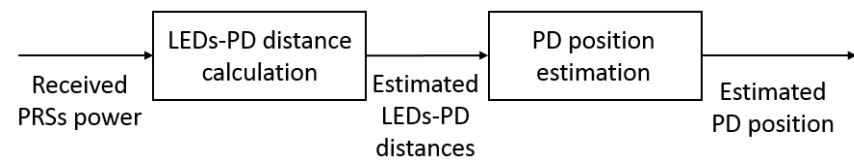

Fig. 2. 2D RSS-TL positioning algorithm.
As shown in Fig. 2, the 2D RSS-TL system includes two steps. First, we should estimate the distance $d_{j}$ from the target PD to each LED $j \in[1, J]$ by using the received PRSs signal power $\mathrm{Pr}_{j}$. According to the Optical Wireless Channel model (OWC) [12], this distance can be computed by

$$
d_{j}=\left(\frac{(m+1) A_{P D} h^{m+1}}{2 \pi} \frac{P t_{j}}{P r_{j}}\right)^{\frac{1}{m+3}}, \quad j \in[1, J]
$$

Second, with the estimated distance vector $\left[d_{j}\right]_{1 \leq j \leq J}^{T}$ and the LED coordinates vector $\left[\left(x_{L E D_{j}}, y_{L E D_{j}}\right)\right]_{1 \leq j \leq J}^{T}$, the estimated PD position $\hat{\boldsymbol{X}}_{P D}=\left(\hat{x}_{P D}, \hat{y}_{P D}\right)$ can be calculated by solving Eqs. (2).

$$
d_{j}^{2}=\left(\hat{x}_{P D}-x_{L E D_{j}}\right)^{2}+\left(\hat{y}_{P D}-y_{L E D_{j}}\right)^{2}+h^{2}, \quad j \in[1, J]
$$

Unfortunately, due to the interference power (noise or reflection powers), there is no common solution to Eq. (2). A least square (LS) estimator will be applied to estimate an optimized PD position. Defining matrices $A$ and $B$ as follows respectively,

$$
\begin{gathered}
\boldsymbol{A}=\left[\begin{array}{cc}
x_{L E D_{2}}-x_{L E D_{1}} & y_{L E D_{2}}-y_{L E D_{1}} \\
x_{L E D_{3}}-x_{L E D_{1}} & y_{L E D_{3}}-y_{L E D_{1}} \\
\vdots & \vdots \\
x_{L E D_{J}}-x_{L E D_{1}} & y_{L E D_{J}}-y_{L E D_{1}}
\end{array}\right]_{2}^{2} x_{L E D_{1}}-y_{L E D_{1}}^{2} \\
\boldsymbol{B}=\frac{1}{2}\left[\begin{array}{c}
d_{1}^{2}-d_{2}^{2}+x_{L E D_{2}}^{2}+y_{L E D_{2}}^{2}-x_{L E D_{1}}^{2}-y_{L E D_{1}}^{2} \\
d_{1}^{2}-d_{3}^{2}+x_{L E D_{3}}^{2}+y_{L E D_{3}}^{2}-x_{L E D_{1}}^{2} \\
\vdots \\
d_{1}^{2}-d_{J}^{2}+x_{L E D_{J}}^{2}+y_{L E D_{J}}^{2}-x_{L E D_{1}}^{2}-y_{L E D_{1}}^{2}
\end{array}\right]
\end{gathered}
$$

then the estimated PD position can be obtained by Eq. (4).

$$
\hat{\boldsymbol{X}}_{P D}=\left(\boldsymbol{A}^{T} \boldsymbol{A}\right)^{-1} \boldsymbol{A}^{T} \boldsymbol{B}
$$

The RSS-TL algorithm is only based on the LOS PRSs power. According to the Optical Wireless Channel model (OWC) [12], however the received PRS power of $j^{t h}$ LED $\left(P r_{j}\right)$ consists of the LOS signal power $\left(P r_{j}^{l o s}\right)$, NLOS signal power $\left(\operatorname{Pr}_{j}^{\text {nlos }}\right)$ as well as the noise $\left(\operatorname{Pr}_{j}^{n}\right)$. The noise involves shot noise and thermal noise which are dependent on the received light power and the temperature, respectively. Both noises are considered as an Additive White Gaussian Noise (AWGN).

$$
\operatorname{Pr}_{j}=P r_{j}^{l o s}+P r_{j}^{n l o s}+P r_{j}^{n} \quad j \in[1, J]
$$

Since the reflection is the most important and common NLOS propagation for indoor environment, the NLOS PRSs is represented by the reflection PRSs. Both reflection and noise will create an error for distance estimation and affect the positioning accuracy. To show this negative impact, we conduct simulations in a common indoor environment: Room size $5 \mathrm{~m} \times 5 \mathrm{~m} \times 3 \mathrm{~m}$ with 4 LEDs, temperature $20^{\circ} \mathrm{C}$, Ambient light $200 \mathrm{~lx}$ [16], and reflection factor of Walls 30\% [17]. The distribution of positioning accuracy is plotted in Fig. 3. In the center area, the 2D RSS-TL algorithm can achieve a good positioning accuracy within $10 \mathrm{~cm}$. In the corner area, with the decrease of LOS PRS power and the increase of reflection PRS power, the positioning error can be as large as 
1 meter. Meanwhile, the reflection PRSs powers are not fixed for the entire room due to the diversity of indoor structure. The reflection light may come from walls, floor, obstacles or humans.

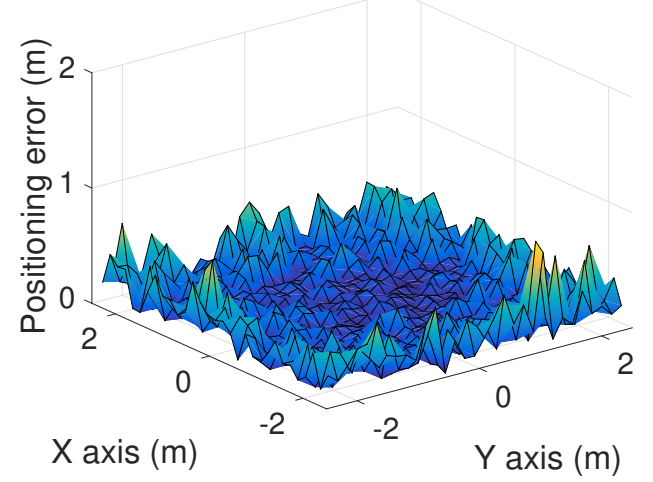

(a)

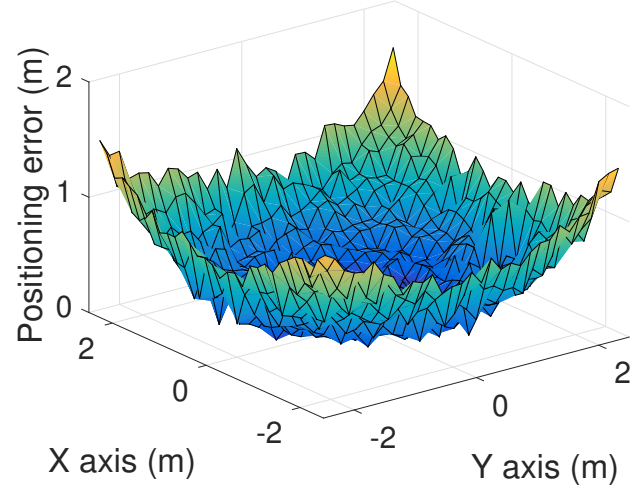

(b)

Fig. 3. Influence of noise (a) and reflection (b) on positioning accuracy for RSS-TL algorithm.

\section{NLOS-AWARE ENHANCED FINGERPRINT RSS TRILATERATION (EFP-RSS-TL) POSITIONING ALGORITHM}

In order to eliminate this negative impact of NLOS light signal and improve the positioning accuracy, we propose an enhanced fingerprint RSS trilateration positioning algorithm (EFP-RSS-TL). Our proposal leverages an enhanced fingerprint (EFP) database and K-Nearest Neighbors (K-NN) method to estimate the received LOS PRSs power as precisely as possible. The framework of our proposal is depicted in Fig. 4, which contains two phases: Offline (database creation) and online (positioning computation).

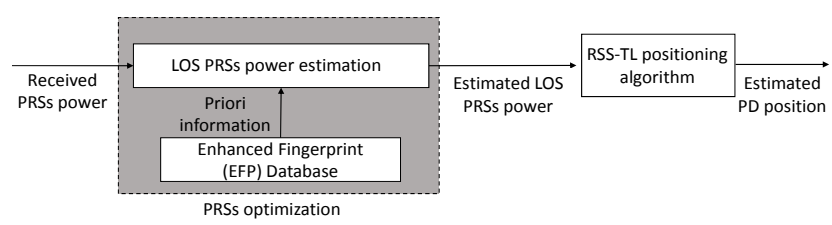

Fig. 4. The framework of our proposal EFP-RSS-TL.

\section{A. The offline phase: Database creation}

In this phase, we construct beforehand an enhanced fingerprint (EFP) database, which includes three matrices.

$$
\boldsymbol{D} \boldsymbol{B}_{E F P}=\left\{\boldsymbol{S}, \tilde{\boldsymbol{P r}}^{\text {los }}, \tilde{\boldsymbol{Q}}\right\}
$$

where $\boldsymbol{S}$ is the matrix of VL-IPS setting parameters, $\tilde{\boldsymbol{P}}^{\text {los }}$ represents the matrix of the reference LOS PRSs power, and $\tilde{Q}$ is the matrix of reference PRSs quality.

1) VL-IPS Setting Parameters Matrix: The matrix $S$ records the positioning related VL-IPS parameters for each LED $j$, such as its transmitted PRSs power $\left(P t_{j}\right)$, horizontal position coordinates $\left(x_{L E D_{j}}, y_{L E D_{j}}\right)$, PRSs frequency $f_{j}$, lambertian factor $m$, effective area of photodiode $\left(A_{P D}\right)$, the responsivity of photodiode $\left(R_{P D}\right)$, as well as the vertical distance $h$.

$$
\boldsymbol{S}=\left[\left(P t_{j}, x_{L E D_{j}}, y_{L E D_{j}}, f_{j}, m, h, A_{P D}, R_{P D}\right)\right]_{1 \leq j \leq J}
$$

2) Reference LOS PRSs Power Matrix: In order to calculate the reference LOS PRSs power, we predetermine $R$ PD positions (e.g., a grid) and select them as reference points in the room. An example is given in Fig. 5. Let $\left(x_{r}, y_{r}\right)$ be the

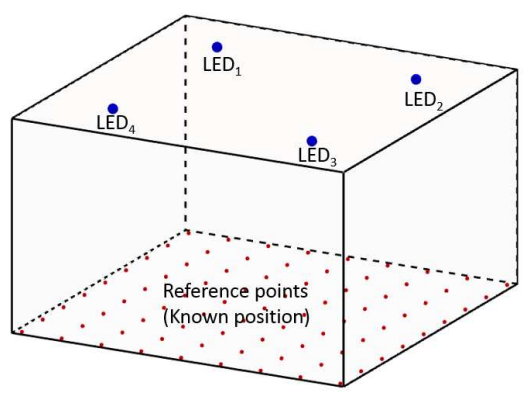

Fig. 5. Example of reference points.

coordinates of the $r$-th reference point, then the distance $d_{j, r}$ to the $j$-th LED can be expressed by Eq. (8)

$$
d_{j, r}=\sqrt{\left(x_{L E D_{j}}-x_{r}\right)^{2}+\left(y_{L E D_{j}}-y_{r}\right)^{2}+h^{2}}
$$

According to the OWC model [12], the received LOS PRSs power $\tilde{\operatorname{Pr}}{ }_{j, r}^{l o s}$ at the $r$-th reference point and from the $j$-th LED can be calculated by

$$
\tilde{P r}_{j, r}^{l o s}=\frac{(m+1) A_{P D} h^{m+1} P t_{j}}{2 \pi d_{j, r}^{m+3}}
$$

This then constitutes the matrix

$$
\tilde{\boldsymbol{P r}}^{\text {los }}=\left[\tilde{P} r_{j, r}^{l o s}\right]_{1 \leq j \leq J, 1 \leq r \leq R}
$$

3) Reference PRSs quality matrix: The PRS quality is defined by the ratio of received LOS PRS power with respect to the received PRS power. If this ratio is one, it means there has no NLOS PRSs power in received PRSs power. For the $j$-th LED and the $r$-th reference point, the values of $\tilde{P}_{j, r}^{l o s}$ have already been saved in the reference LOS PRSs power matrix. Meanwhile, by real measurement, we can get the real 
received PRSs power $\tilde{P} r_{j, r}$. Consequently, wen can calculate the reference PRS quality $\tilde{Q}_{j, r}$ by

$$
\tilde{Q}_{j, r}=\frac{\tilde{P}_{j, r}^{\text {los }}}{\tilde{P r}_{j, r}}, \quad j \in[1, J] ; r \in[1, R]
$$

Finally, we can obtain the reference PRSs quality matrix

$$
\tilde{\boldsymbol{Q}}=\left[\tilde{Q}_{j, r}\right]_{1 \leq j \leq J, 1 \leq r \leq R}
$$

\section{B. The online phase: positioning}

The objective of this phase is to estimate the coordinates of the target PD. As we have shown in the previous section, the NLOS signal is the key factor degrading the positioning accuracy. In light of this, we propose a novel method to estimate the received LOS PRSs power from each LED as precisely as possible by eliminating the negative impact of NLOS light. To achieve this goal, we create an adaptive-size EFP database and make use of $K$-Nearest Neighbors ( $K$-NN) method. The procedure of this phase is shown in Fig. 6, which has five steps.

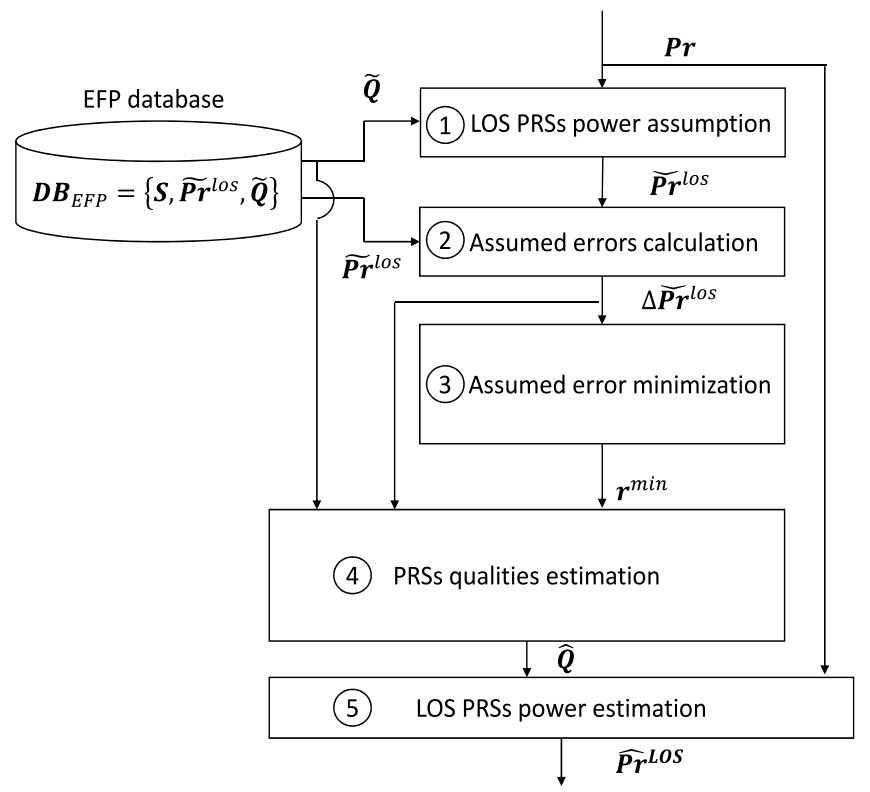

Fig. 6. Process of EFP-RSS-TL algorithm.

With the detected received PRSs power $\boldsymbol{P r}$ at the target PD side, our proposal will compute a set of assumed received LOS PRSs powers by using the reference PRSs quality matrix $\tilde{Q}$. We denote the assumed received LOS PRSs power from $j$-th LED at the $r$-th reference point as $\breve{P r_{j, r}}$, which is expressed by Eq.(13).

$$
\breve{P r} r_{j, r}^{l o s}=\tilde{Q}_{j, r} P r_{j}, j \in[1, J] ; r \in[1, R]
$$

In the second step, we compute the assumed error $\Delta P r_{j, r}^{\text {los }}$ for each reference point, which is defined as the difference between the assumed LOS power and reference LOS power.

$$
\Delta P r_{j, r}^{l o s}=\breve{P r}_{j, r}^{l o s}-\tilde{P r}_{j, r}^{l o s}, j \in[1, J] ; r \in[1, R]
$$

After that, we need to calculate the sum of the assumed error of $J$ LEDS for each reference point. The $K$ reference points with the lowest square error sum will be selected as $K$-NN reference points noted as $\boldsymbol{r}^{\text {min }}=\left[r_{k}^{\text {min }}\right]_{1 \leq k \leq K}$, i.e.,

$$
r_{k}^{\text {min }}=\arg \min _{r \notin \boldsymbol{r}_{\text {min }}}\left(\sum_{j=1}^{J} \Delta \operatorname{Pr}_{j, r}^{2}\right), \quad k \in[1, K]
$$

Given these $K$-NN reference points, we next compute the approximated PRS quality for each LED by a weighted $K$ NN method. To this end, we define $w_{j, k}$ as the weight factor for $j$-th LED, which is given by

$$
w_{j, k}=\frac{1}{\Delta \operatorname{Pr}_{j, r_{k}^{2 \log }}^{\operatorname{los}}}, \quad j \in[1, J], k \in[1, K]
$$

Thus, we can obtain an approximated PRS quality $\hat{Q}_{j}$ as follows

$$
\hat{Q}_{j}=\frac{1}{\sum_{k=1}^{K} w_{j, k}} \sum_{k=1}^{K}\left(w_{j, k} \tilde{Q}_{j, r_{k}^{\text {min }}}\right), \quad j \in[1, J]
$$

At last, the approximated PRS quality vector $\hat{\boldsymbol{Q}}=\left[\hat{Q}_{j}\right]_{1 \leq j \leq J}$ can be used to eliminate the NLOS power. As a result, the LOS PRSs power vector $\hat{\boldsymbol{P r}}^{\text {los }}=\left[\hat{\mathrm{P}}_{j}{ }^{l o s}\right]_{1 \leq j \leq J}$ can be precisely estimated by

$$
\hat{P}_{j}^{\text {los }}=\hat{Q}_{j} P r_{j} \quad j \in[1, J]
$$

After the LOS PRSs power optimization using the EFP method, the PD position can be computed by substituting $\left[P r_{j}\right]_{1 \leq j \leq J}$ with $\left[\hat{P r}_{j}^{\text {los }}\right]_{1 \leq j \leq J}$ in Eqs.(1)-(4).

To summarize, the novelty of our proposal lies at the precise estimation of the LOS PRSs power, which is critical for the positional accuracy. The traditional RSS-TL algorithm uses directly the received PRSs power for computing the target PD position, which contains both the LOS and NLOS PRSs powers. Although RSS-FP uses a database, only the absolute values of PRS powers are stored for positioning. Thus, it is also sensitive to environment changes. Differently, our algorithm proposes to create an a-priori EFP database to store the PRSs quality ratio. As the LOS PRS quality ratio is stored, we can estimate the LOS PRS power as precisely as possible in different light environments and resolve the positioning equation to localize the target. Thus, our proposal enables to distinguish these two powers and eliminate in turn the negative influence of NLOS signal.

\section{EXPERIMENTAL VALIDATION}

In order to validate the proposed EFP-RSS-TL method, we built a real VL-IPS platform. Real experiments were then conducted in a room of size $2.7 \mathrm{~m}$ (width) $\times 4.7 \mathrm{~m}$ (length) $\times$ $2.38 \mathrm{~m}$ (height), which is shown in Fig. 7. Three LEDs are installed on the ceiling and used for positioning. The receiver is posed on the floor with height $0.187 \mathrm{~m}$. A positioning area of $2 \mathrm{~m} \times 1 \mathrm{~m}$ is located at the left side of the room. The LEDs and PD parameters are given in Tab. I. Three LEDs (Spot LED MR16 4W-12V) emit 500, 700, $900 \mathrm{kHz}$ sine waves as PRSs, respectively. The receiver PD (Hamamatsu APD C12702-12) will detect the received PRSs and analyze it in Lumen.

Our experimental validation involves two parts: data acquisition and performance validation. The performance of our proposal is then evaluated in terms of positioning accuracy, system robustness to environment and system complexity. 


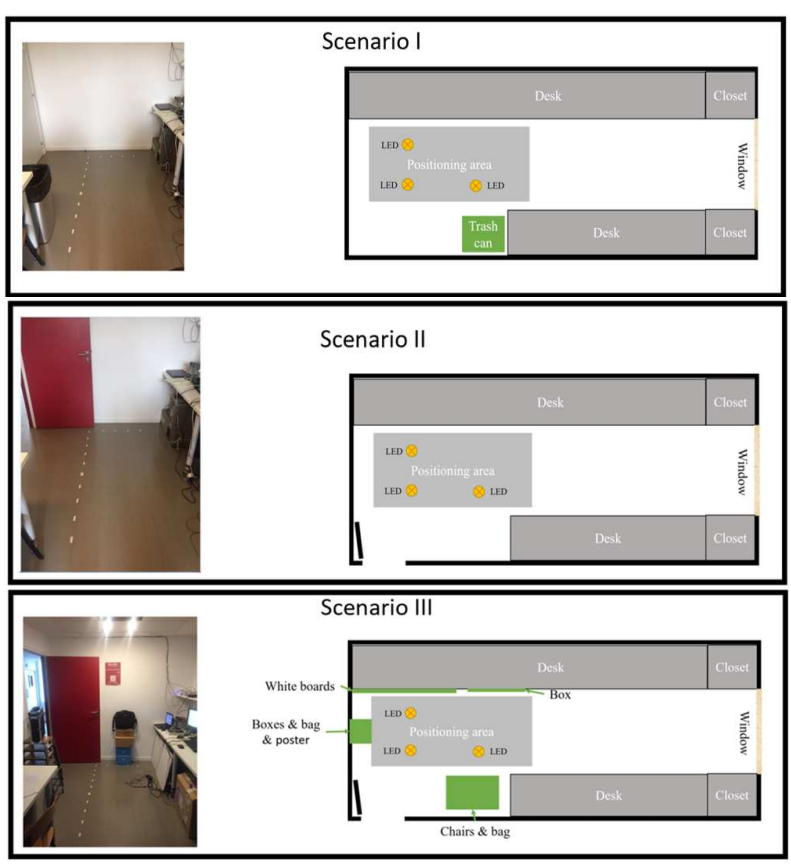

Fig. 7. Real VL-IPS platform and scenarios.

TABLE I

REAL VL-IPS PLATFORM PARAMETERS

\begin{tabular}{|c|c|}
\hline Parameters & Typical values \\
\hline Positioning area $(\mathrm{L} * \mathrm{~W})$ & $2 \mathrm{~m} \times 1 \mathrm{~m}$ \\
Height of PD & $0.175 \mathrm{~m}$ \\
Position of LEDs & $(-0.5,0.25,2.4)(-0.5,0.6,2.4)(0.5,0.25,2.4)$ \\
Transmitted PRSs power & $3.01 \mathrm{~m}$ \\
Lambertian factor of LED & 1.56 \\
Gain of optical filter & 1 \\
Gain of optical concentrator & 1 \\
Effective area of PD & $7 \mathrm{E}-6 \mathrm{~m}^{2}$ \\
\hline
\end{tabular}

\section{A. Data acquisition}

In order to create the EFP database, we first make some measurements and collect data in different scenarios at different time of a day. As shown in the Fig. 7, three scenarios are considered. For scenario I, the door is closed and a trash can is posed near the desk. For scenario II, the door is opened and the trash can is removed. In scenario III, some black bags, chairs, boxes and white board are put in the different area of the room. For each scenario, three measurements are taken at different time of a day, as shown in Tab. II. During the daytime $10-17 \mathrm{~h}$, the room is occupied by $2-4$ persons and the presence of human will cause some random interference on lighting environment.From $18 \mathrm{~h}$ to $20 \mathrm{~h}$, the room is vacant and the lighting environment is more stable.

In the experiment room, we predetermined $21 \times 11$ sampling points and recorded their coordinates. We then computed their distances to each LED by Eq. (8). These points were then used either as reference points for creating the EFP database or used for evaluating the positioning error. For each point and lighting environment, we measure also the PRSs power received at the target PD from each LED. Meanwhile, we can calculate the estimated reference LOS PRSs power of a reference point by Eq. (9). With these measurements, we obtain the reference
TABLE II

DEFINITION OF EXPERIMENT ENVIRONMENT

\begin{tabular}{|c|c|c|}
\hline Scenario & Lighting environment & Description \\
\hline \multirow{3}{*}{ Scenario I } & E1 & Time: $18 \mathrm{~h}-20 \mathrm{~h}$ (vacant room) \\
& E2 & Time: $10 \mathrm{~h}-12 \mathrm{~h}$ (Occupy room with 2-4 persons) \\
& E3 & Time: $12 \mathrm{~h}-17 \mathrm{~h}$ (Occupy room with 2-4 persons) \\
\hline \hline \multirow{3}{*}{ Scenario II } & E4 & Time: $18 \mathrm{~h}-20 \mathrm{~h}$ (vacant room) \\
& E5 & Time: $10 \mathrm{~h}-12 \mathrm{~h}$ (Occupied room with 2-4 persons) \\
\hline \multirow{3}{*}{ Scenario III } & E6 & Time: $12 \mathrm{~h}-17 \mathrm{~h}$ (Occupied room with 2-4 persons) \\
& E7 & Time: $18 \mathrm{~h}-20 \mathrm{~h}$ (vacant room) \\
& E8 & Time: $10 \mathrm{~h}-12 \mathrm{~h}$ (Occupied room with 2-4 persons) \\
& E9 & Time: $12 \mathrm{~h}-17 \mathrm{~h}$ (Occupied room with 2-4 persons) \\
\hline
\end{tabular}

PRSs quality matrix according to Eq. (11). Finally, we finish the creation of the EFP database by recording these data.

\section{B. Algorithm performance validation}

In order to validate our proposal, we compare our proposal (EFP-RSS-TL algorithm) with two benchmark algorithms, namely RSS-TL and RSS-FP. Three series of evaluations are conducted: positioning accuracy, system robustness to environment, as well as the system complexity.

1) Positioning accuracy: We plot the distribution of positioning error for the three algorithms in Fig. 8. The constructed EFP database contains 15 reference points. Both the reference data (EFP database) and the positioning data (PRSs power received at the target $\mathrm{PD}$ ) are acquired in the same environment E1. We observe that the RSS-TL has significant positioning error especially in the room corners, although it obtains a good positioning performance in the room center. This can be explained by its sensitivity due to the light reflection. The RSSFP can diminish the impact of reflection but its positioning error can be as large as $50 \mathrm{~cm}$ in some area. Because the performance of RSS-FP is restricted by limited reference point number and its distribution. In contrast, our proposal achieves an average positioning error of $6.19 \mathrm{~cm}$ for the entire room. Compared with $26.3 \mathrm{~cm}$ of RSS-TL and $15.12 \mathrm{~cm}$ of RSS-FP, our proposal reduces $72.7 \%$ and $59 \%$ of positioning error, respectively. This result can be interpreted as follows. By using the EFP database to record the LOS Power ratio at the reference points, our algorithm enables to eliminate the impact of reflection and NLOS power. As a result, our EFP-RSS-TL permits to cut significantly the positioning error in the corner while keeping a high positing accuracy in the room center.

Besides, we repeat the same analysis for nine lighting environments (E1-E9) specified in Tab. II. The average positioning error of the entire room is presented in Tab. III. We can find that our proposal can reduce the impact of reflection and improve the positioning accuracy in all lighting environments.

2) System Robustness to environments: In the previous analysis, the positioning evaluation and the measurements for EFP database creation were done in the same lighting environment. In reality, the environment is changeable in any time. Consequently, it is difficult to guarantee the same lighting environment for them. Thus, we will evaluate the system robustness to lighting environment change. In this part, only the algorithms based on database are considered: RSS-FP and EFP-RSS-TL. We created nine different EFP databases $\left(D B_{1}-D B_{9}\right)$, and each database $D B_{i}$ records the data of 

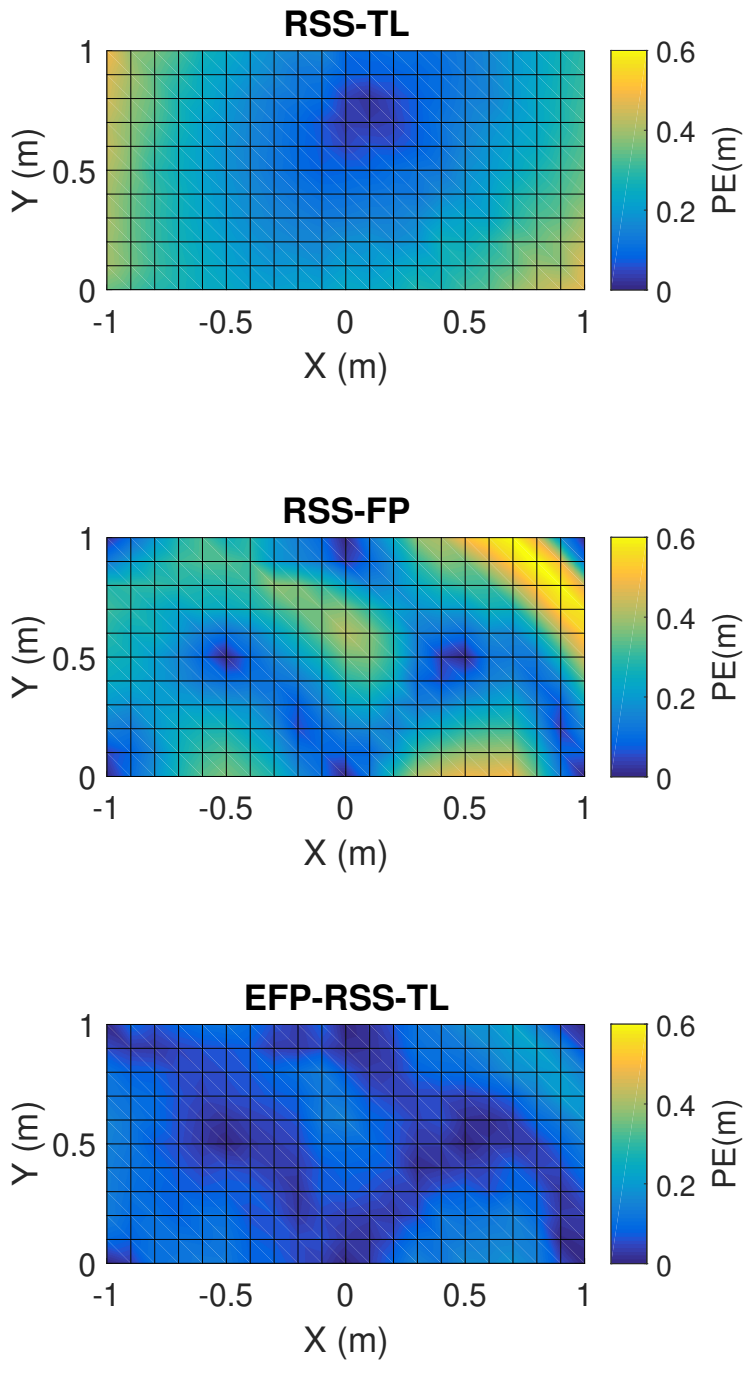

Fig. 8. Distribution of positioning error (PE) with different algorithms.

TABLE III

AVERAGE POSITIONING ERROR IN DIFFERENT LIGHTING ENVIRONMENTS

\begin{tabular}{|c|ccccc|}
\hline & \multicolumn{5}{|c|}{ Average PE (cm) } \\
& E1 & E2 & E3 & E4 & E5 \\
\hline RSS-TL & 26.3 & 27.2 & 28.7 & 22.3 & 23.2 \\
RSS-FP & 15.1 & 16.6 & 17.0 & 15.8 & 17.3 \\
EFP-RSS-TL & 6.2 & 12.2 & 14.5 & 5.5 & 12.2 \\
\hline & \multicolumn{5}{|c|}{ Average PE (cm) } \\
\hline & E6 & E7 & E8 & E9 \\
\hline RSS-TL & 25.5 & 21.8 & 23.9 & 24.9 \\
RSS-FP & 18.1 & 15.1 & 17.8 & 19.4 & \\
EFP-RSS-TL & 14.5 & 5.1 & 10.0 & 14.4 & \\
\hline
\end{tabular}

15 reference points acquired in the corresponding lighting environment $E_{i}$. The databases creation and positioning tests were conducted in two unrelated environments. Thus, $9 \times 9$ positioning tests in total were done for each algorithm. Fig. 9 plots the average PE for each test and presents also the distribution of average PE among four error ranges. According to the results, our proposal (EFP-RSS-TL) has a probability of $20 \%$ to achieve a PE less than $10 \mathrm{~cm}$ and only $16 \%$ to have a PE in the range $30-40 \mathrm{~cm}$. While for RSS-FP, $41 \%$ of the tests fall into the PE range $30-40 \mathrm{~cm}$, and no test has a PE less than $10 \mathrm{~cm}$. Thus, we can say that our proposal is less sensitive to lighting environment change.

3) System complexity: The number of reference points has a significant impact on the system complexity, for both database creation and positioning computation. In this part, we first assert the computing time and storage size when changing the number of reference points from 6 to 66 for E1 in Fig. 10. One can find that the PE of RSS-TL algorithm is fixed at $27.6 \mathrm{~cm}$, which does not depend on the number of reference points. While the positioning error will be reduced for the other two algorithms, when the number of reference points increases. It should also be noted that our proposal results in a lower installation \& maintenance cost for VL-IPS, because it cuts significantly the number of reference points required to achieve a same PE. For example, in order to achieve a PE of $10 \mathrm{~cm}$, our proposal only uses 8 reference points in the EFP database, while 7 times more reference points are required for RSS-FP.

Even though our proposal requires fewer reference points, the EFP database is more complex than that of RSS-FP. Thus, we also plot the average PE with respect to the computing time and the database size in Fig. 11. To get a PE of $10 \mathrm{~cm}$, it is shown that the database size used by RSS-FP algorithm is almost the twice of that used by our proposal. Thus our proposal needs a smaller storage space. But we can also observe that our proposal requires more computing time than RSS-FP, which however is still in the order of $0.1 \mathrm{~ms}$. Because, compared to the RSS-FP algorithm, our algorithm needs to do some additional computation, i.e., compute the estimated LOS Power and resolve Eqs.(1)-(4) for positioning the target.

\section{CONCLUSIONS}

In this paper, we propose a novel VLC-based indoor localization algorithm, namely EFP-RSS-TL. By leveraging a database to store the line-of-sight light power quality ratios, our algorithm is able to eliminate the negative impact of light reflection. The performance of our algorithm is validated by real experiments on a real VL-IPS testbed. It is shown that our algorithm is not only able to improve significantly the positioning accuracy, but also more robust to lighting environment change compared to its counterparts in the literature.

\section{REFERENCES}

[1] Y. Qiu, H.-H. Chen, and H.-H. Chen, "Channel modeling for visible light communications-a survey." Wireless Communications Mobile Computing, vol. 16, no. 14, 2016.

[2] W. Wu, F. Zhou, and Q. Yang, "Adaptive network resource optimization for heterogeneous vlc/rf wireless networks," IEEE Transactions on Communications, vol. 66, no. 11, pp. 5568-5581, Nov 2018.

[3] H. Huang, L. Feng, P. Guo, and A. Y. G. Ni, "Iterative positioning algorithm to reduce the impact of diffuse reflection on an indoor visible light positioning system," Optical Engineering, vol. 55, p. 066117, 06 2016.

[4] J. Luo, L. Fan, and H. Li, "Indoor positioning systems based on visible light communication: State of the art." IEEE Communications Surveys Tutorials, vol. 19, no. 4, 2018. 

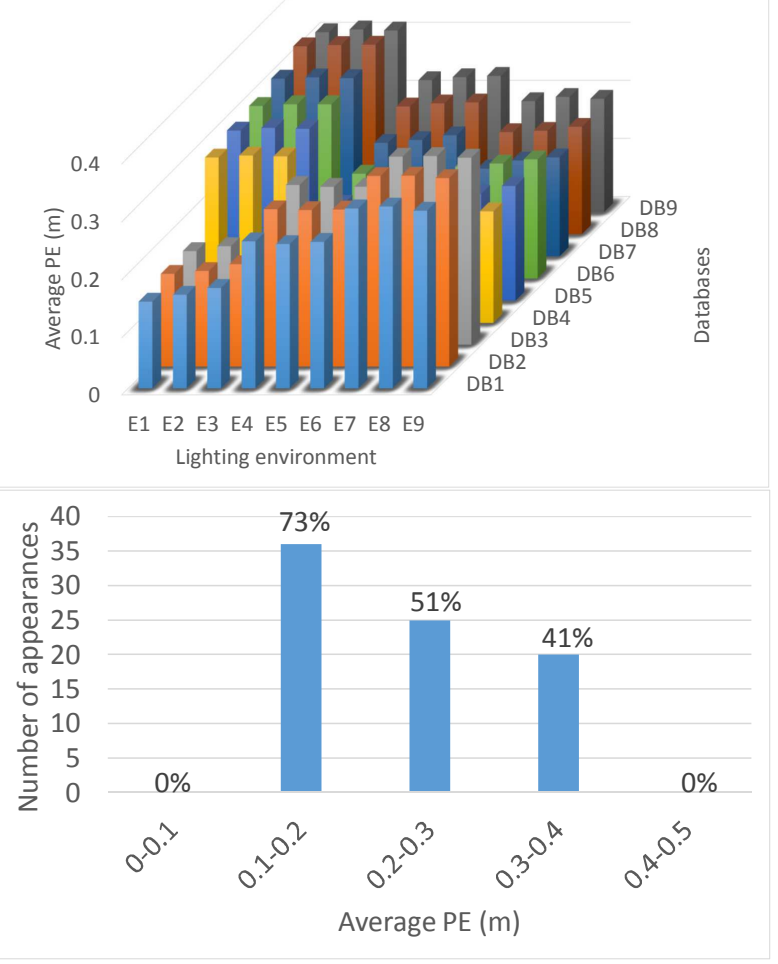

(a) RSS-FP
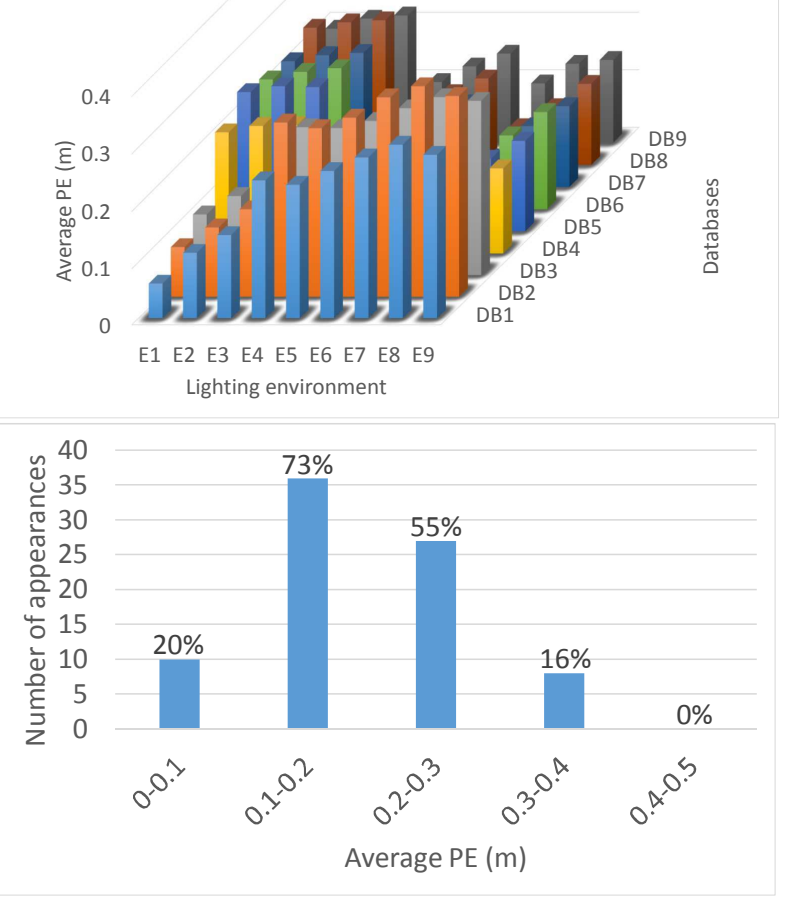

(b) EFP-RSS-TL

Fig. 9. System robustness to different lighting environments.

[5] T. Q. Wang, Y. A. Sekercioglu, A. Neild, and J. Armstrong, "Position accuracy of time-of-arrival based ranging using visible light with application in indoor localization systems." Journal of Lightwave Technology,

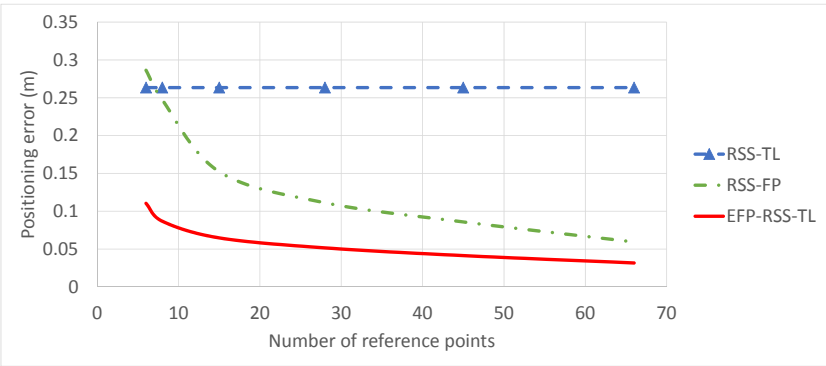

Fig. 10. Average PE vs. number of reference points.
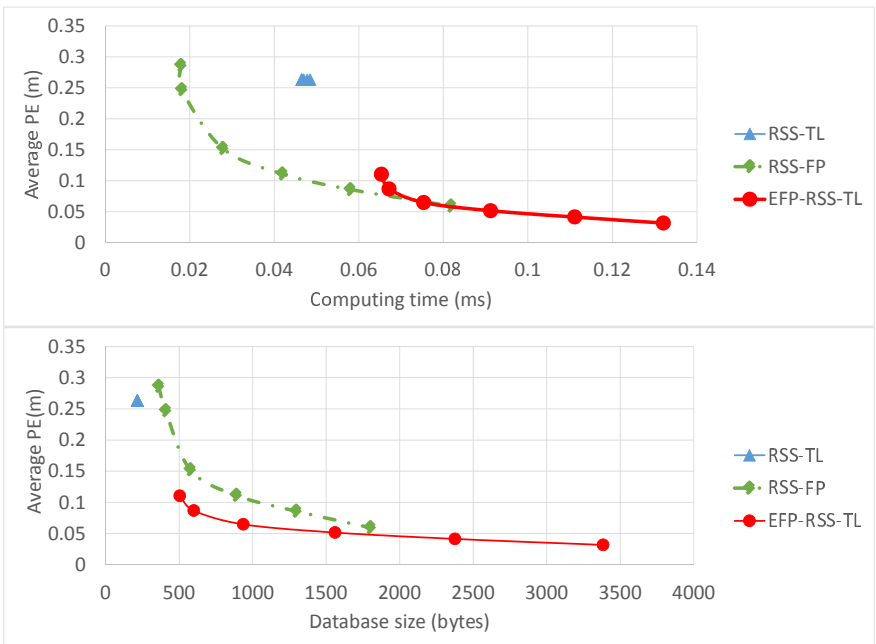

Fig. 11. Computing time \& database size Vs. PE

vol. 31, no. 20, pp. $3302-3308,2013$.

[6] S.-Y. Jung, S. Hann, and C.-S. Park, "Tdoa-based optical wireless indoor localization using led ceiling lamps." IEEE Transactions on Consumer Electronics, vol. 57, no. 4, pp. 1592 - 1597, 2011.

[7] H.-S. Kim, D.-R. Kim, S.-H. Yang, Y.-H. Son, and S.-K. Han, "An indoor visible light communication positioning system using a rf carrier allocation technique." Journal of Lightwave Technology, vol. 31, no. 1, pp. 134 - 144, 2013.

[8] M. Yasir, S.-W. Ho, and B. N. Vellambi, "Indoor position tracking using multiple optical receivers." Journal of Lightwave Technology, vol. 34, no. 4, pp. 1166 - 1176, 2016.

[9] A. Naz, H. M. Asif, T. Umer, and B.-S. Kim, "Pdoa based indoor positioning using visible light communication." IEEE Access, vol. 6, pp. 7557-7564, 2018.

[10] S. Jung, S. Hann, S. Par, and C. Park, "Optical wireless indoor positioning system using light emitting diode ceiling lights." Microwave and Optical Technology Letters, vol. 54, no. 7, pp. 1622-1626, 2012.

[11] Y.-S. Kuo, P. Pannuto, K.-J. Hsiao, and P. Dutta, "indoor positioning with mobile phones and visible light." MobiCom '14 Proceedings of the 20th annual international conference on Mobile computing and networking, pp. 447-458, 2014.

[12] Z. Ghassemlooy, W. Popoola, and S. Rajbhandari, Optical Wireless Communications. CRC Press, 2012.

[13] W. Gu, M. A. Kashani, and M. Kavehrad, "Multipath reflections analysis on indoor visible light positioning system." IEEE Globecom, 2015.

[14] S. Subedi and J.-Y. Pyun, "Practical fingerprinting localization for indoor positioning system by using beacons," Hindawi, J. Sens, pp. 1-16, 2017.

[15] X. Guo, L. Chu, B. Li, B. Xu, Q. Wan, and Y. Shen, "A robust vector matching localization approach based on multiple channels ssd fingerprinting of zigbee networks," Prog. Electro magn, vol. 144, pp. 133-140, 2014.

[16] V. Group, Daylight, Energy and Indoor Climate Basic Book, 2014.

[17] EngineeringToolBox, "Materials - light reflecting factors," https://www. engineeringtoolbox.com/light-material-reflecting-factor-d_1842.html, accessed Feb. 1, 2019. 